UCRL-ID-125394

\title{
The Use of Implicit Monte Carlo Radiation Transport with Hydrodynamics and Compton Scattering
}

J. A. Fleck, Jr.

DEC $O 91998$

OSTI

March, 1971

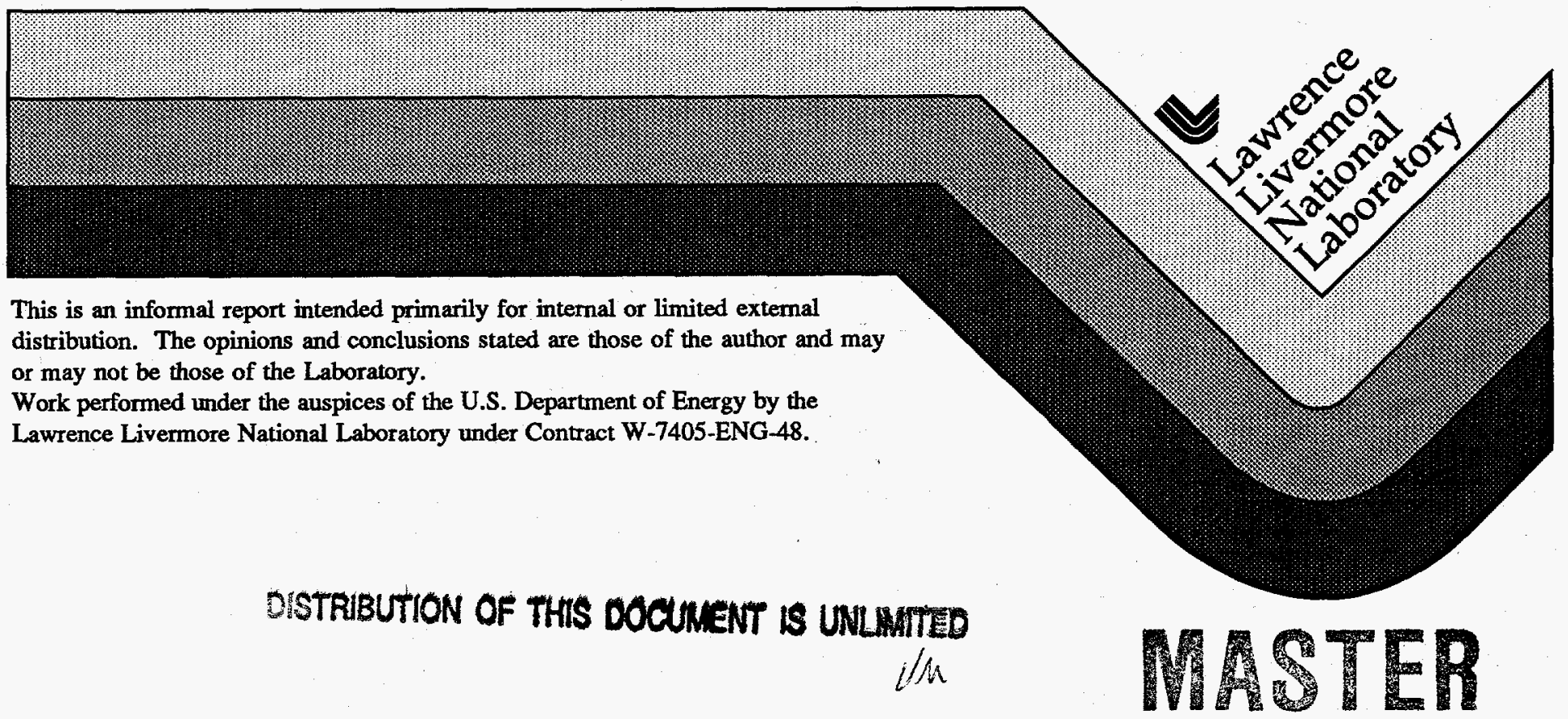




\section{DISCLAIMER}

This document was prepared as an account of work sponsored by an agency of the United States Government. Neither the United States Government nor the University of California nor any of their employees, makes any warranty, express or implied, or assumes any legal liability or responsibility for the accuracy, completeness, or usefulness of any information, apparatus, product, or process disclosed, or represents that its use would not infringe privately owned rights. Reference herein to any specific commercial product, process, or service by trade name, trademark, manufacturer, or otherwise, does not necessarily constitute or imply its endorsement, recommendation, or favoring by the United States Government or the University of California. The views and opinions of authors expressed herein do not necessarily state or reflect those of the United States Government or the University of California, and shall not be used for advertising or product endorsement purposes.

This report has been reproduced directly from the best available copy.

Available to DOE and DOE contractors from the Office of Scientific and Technical Information

P.O. Box 62, Oak Ridge, TN 37831

Prices available from (615) 576-8401, FTS 626-8401

Available to the public from the

National Technical Information Service

U.S. Department of Commerce

5285 Port Royal Rd.

Springfield, VA 22161

Work performed under the auspices of the U.S. Department of Energy by Lawrence Livermore National Laboratory under Contract W-7405-ENG-48. 


\section{DISCLAMMER}

Portions of this document may be illegible in electronic image products. Images are produced from the best available original document. 


\section{Inavromce IRadiation Iaboxatoxy UNIVERSITY OF CALIFORHIA \\ LIVERMORE}

THE USE OF TMPLICIT MONIE CARLO RADIATION

TRANSPORT WITH HYDRODYNAMICS AND COMPTON SCATTERTNG

J. A. Fleck, Jr.

March, 1971 
THE USE OF IMPLICIT MONTE CARLO RADIATION

TRANSPORT WITH HYDRODYNAMICS AND COMPTON SCATTERING

J. A. FLECK, JR.

March, 1971

\section{INTRODUCTION}

The purpose of this note is to show that the combination of implicit radiation transport and hydrodynamics, Compton scattering, and any other energy transport or exchange mechanism can be simply carried out by a "splitting" procedure. This means that the contributions to material energy exchange can be reckoned separately for hydrodynamics, radiation transport without scattering, Compton scattering, plus any other possible energy exchange mechanism. The radiation transport phase of the calculation would of course be implicit and would be camied out in exactly the manner described in UCRL $-7294^{1}$, but the hydrodynamics and Compton portions would not, leading to possible time step controls. In any case, the time step restrictions which occur on radiation transfer due to lange Planck mean absorption cross-sections would not occur.

\section{EQUATIONS}

The equations which govern the radiation transport are $\frac{I}{C} \frac{\partial I_{\nu}}{\partial t}+\mu \frac{\partial I_{\nu}}{\partial x}+\left(\sigma_{\nu}+\sigma_{\nu}{ }^{C}\right) I_{\nu}=\int \sigma^{C}\left(\mu^{\prime}, \nu^{\prime} \rightarrow \mu, \nu\right) I\left(\mu^{\prime}, \nu^{\prime}\right) \frac{\nu}{\nu^{\prime}} d \mu^{\prime} d \nu^{\prime}+\frac{1}{2} C \sigma{ }_{\nu} B_{\nu}$ 


$$
\frac{\partial u_{m}}{\partial t}=\iint \sigma_{\cdot v} I_{\nu} d v d \mu-c \int \sigma_{v} B_{v} d v+s
$$

In Equation (la) $\sigma_{v}$ is the frequency dependent absorption cross-section, $\sigma_{\nu}^{C}$ is the total frequency dependent compton scattering cross-section, and $\sigma^{C}\left(\mu ; \nu^{\prime}+\mu, \nu\right)$ is the cross-section for Compton scattering from direction $\mu^{\prime}$ and frequency $\nu^{\prime}$ to direction $\mu$ and frequency $v$. In Eq. (Ia) stimulated or induced scattering contributions have been neglected. In Eq. (Ib) $u_{m}$ represents the matter energy density and $S$ represents the rate of energy exchange to the matter from all sources other than absorption of radiation, e.g. pdv work and compton exchange. For the sake of definitimess we shall assume that the compton energy exchange remains from the previous cycle and that the hydrodynamics and pdv work are computed at the beginning of the current cycle. These restrictions will be removed later.

In order to derive the implicit radiation transfer equations we write Eq. (Ia) as b

$$
\frac{\partial u_{r}}{\partial t}=\beta\left\{\int \sigma_{\nu} I_{\nu} d v d \mu-c \sigma_{p} u_{r}\right\}+\beta S
$$

where

$$
u_{n}=a T^{4},
$$

and

$$
B=\frac{\partial u_{r}}{\partial u_{m}}=\frac{4 a T^{3}}{b} \text {. }
$$

with

$$
b(T)=\frac{\partial u_{m}}{\partial T}
$$


Integrating Eq. (2) from time $t^{n}$ to time $t^{n+1}$ we get

$$
\begin{aligned}
& u_{r}^{n+1}-u_{r}^{n}=\int_{t^{n}}^{t^{n+1}} d t \beta \iint_{v} I_{v} d v d \mu-c \int_{t^{n}}^{t n+1} d t \beta \sigma_{p} u_{r}+\int_{t^{n}}^{t^{n+1}} d t \beta s \\
& u_{r}^{n+1}-u_{r}^{n}=\bar{B} \Delta t\left\{\int \sigma_{v_{v}} d v d \mu-c \bar{\sigma}_{p}\left[\alpha u_{r}^{n+1}+(I-\alpha) \bar{B} \bar{s} \Delta t\right.\right. \\
& \left.+(I-\alpha) u_{r}^{n}\right]
\end{aligned}
$$

In going from Eq. (4a) to Eq. (4b) we have represented the average value of $u_{1}$ under the integral sign in the second right hand member of (4a) as

$$
\bar{u}_{r}=\alpha u_{r}^{n+1}+(I-\alpha)\left[u_{r}^{n}+\bar{B} \bar{S} \Delta t\right]
$$

instead of by means of the more accurate expression (at least when $\alpha=1 / 2$ )

$$
\bar{u}_{r}=\alpha u_{r}^{n+1}+(1-\alpha) u_{r}^{n}
$$

which was used in UCRL-72924. Expression (4c) is employed for fommal reasons which will be apparent shortly. In the completely implicit case $(\alpha=1)$ Eqs. (4c) and (4d) are identical.

We now make the identification

$$
u_{r}^{n^{*}}=u_{r}^{n}+\beta S \Delta t
$$

where, for convenience, average bars have been dropped. Here the superscript $n^{*}$ signifies a time between $t^{n}$ and $t^{n+1}$. Solving Eq. (4b) for $u_{r}^{n+1}$ now yields

$$
u_{r}^{n+1}=\frac{B \Delta t}{1+\alpha \beta c \Delta \sigma_{p}} \int \bar{I}_{v} \sigma_{v} d v d \mu+\left[\frac{1-(1-\alpha) B c \Delta t \sigma_{p}}{1+\alpha \beta c \Delta t \sigma_{p}}\right] u_{r}^{n *}
$$


This is Iike Eq. (1.8) of UCRL-72924 but with the source term eliminated. If $\bar{u}_{r}$ is calculated from

$$
\bar{u}_{n}=\alpha u_{n}^{n+1}+(1-\alpha) u_{n}^{n *}
$$

and substituted into the transport equation (la) the result is

$$
\begin{aligned}
\frac{I}{c} \frac{\partial I_{v}}{\partial x}+\mu \frac{\partial I_{v}}{\partial x}+\left(\sigma_{v}\right. & +\sigma_{\nu} I_{v}=\frac{I}{2} \sigma_{v} b_{v}\left[\frac{\alpha \beta c \Delta t}{I+\alpha \beta c \Delta t \sigma_{p}}\right] \iint \sigma_{v^{\prime}} I_{v}, d v^{\prime} d \mu^{\prime} \\
& +\int \sigma^{c}\left(\mu^{\prime}, v^{\prime} \rightarrow \mu, v\right) I\left(\mu^{\prime}, v^{\prime}\right) \frac{v}{v^{\prime}} d \mu^{\prime} d v^{\prime} \\
& +\frac{1}{2} \frac{c \sigma_{v} b_{v} u^{n^{*}}}{1+\alpha \beta c \Delta t \sigma_{p}},
\end{aligned}
$$

where $b_{v}$ represents a normalized Planck spectrum, and any reference to the source term $S$ is now contained in $u_{r}^{n^{* *}}$. The interpretation of (8) is that the effective absorption cross-section is now

$$
\sigma_{v a}=\sigma_{i v}\left[\frac{1}{1+\alpha \beta c \Delta t \sigma_{p}}\right] \text {, }
$$

the effective scattering cross-section due to absorption and reemission is

$$
\sigma_{\nu s}=\alpha_{\nu}\left[\frac{\sigma^{\alpha \beta c \Delta t}}{1+\alpha \beta c \Delta t \sigma_{p}}\right] \text {, }
$$

and the total effective scattering cross-section is

$$
\sigma_{s}^{T}=\sigma_{v}^{C}+\sigma_{v s}
$$

Equation (8) is assumed valid from time $t^{n}$ to $t^{n+1}$. Thus we now integrate Eq. (8) over angle and frequency to obtain a conservative form of the matter energy density equation. We obtain 


$$
\begin{aligned}
\frac{I}{c} \frac{\partial}{\partial t} \iint d v d \mu I_{v} & +\frac{\partial F}{\partial x}=-\frac{\partial u_{m}}{\partial t}+s=-\left|\frac{\partial u_{m}}{\partial t}\right|_{C}-\iint d v d v \sigma_{v} I_{v} \\
& +\left(\frac{\alpha \beta c \Delta t_{\sigma_{p}}}{I+\alpha \beta C \Delta t \sigma_{p}}\right) \iint d v d \mu \sigma_{v} I_{v}+\frac{c \sigma_{p} u_{p}^{n^{*}}}{I+\alpha \beta c \Delta \sigma_{p}}
\end{aligned}
$$

In $E q .(12)\left(\partial u_{m} / \partial t\right) C^{r e p r e s e n t s ~ t h e ~ e n e r g y ~ e x c h a n g e ~ r a t e ~ f r o m ~ r a d i a t i o n ~}$ to matter due to Compton scattering and is given by

$$
\begin{aligned}
\left|\frac{\partial u_{m}}{\partial t}\right|_{C} & =\int d \mu d v \int \sigma\left(\mu, \nu \rightarrow \mu^{\prime}, v^{\prime}\right) I(\nu, \mu) d v^{\prime} d \mu^{\prime} \\
& -\int d \mu d v \int \sigma\left(\mu^{\prime}, v^{\prime} \rightarrow \mu, v\right) I\left(\nu, \mu^{\prime}\right) \frac{v}{v^{\prime}} d v^{\prime} d \mu^{\prime}
\end{aligned}
$$

Combining terms in Eq. (12) gives

$$
\frac{\partial u_{m}}{\partial t}=s+\left(\frac{\partial u_{m}}{\partial t}\right)_{c}+\frac{1}{1+\alpha \beta c \Delta t \sigma_{p}} \iint d v d \mu \sigma_{v} I_{v}-\frac{c \sigma_{p} u_{r}^{n^{*}}}{1+\alpha \beta c \Delta t \sigma_{p}}
$$

Replacing $\partial u_{m} / \partial t$ by $b(T)(\partial T / \partial t)$ and integrating Eq. (14) from $t^{n}$ to $t^{n+1}$ gives

$$
\begin{aligned}
T^{n+1}=T^{n}+ & b^{-1}\left\{s \Delta t+\left(\left.\frac{\partial u_{m}}{\partial t}\right|_{C} \Delta t+\frac{1}{1+\alpha \beta c \Delta t \sigma_{p}} \int_{t^{n}}^{t^{n+1}} d t \iint d v d_{j} \sigma_{v} I_{v}\right.\right. \\
& \left.-\frac{c \sigma_{p} \Delta t u_{p}^{n^{*}}}{1+\alpha \beta c \Delta t \sigma_{p}}\right\}
\end{aligned}
$$

We can define

$$
T^{n^{*}}=T^{n}+b^{-1} S \Delta t
$$

If the updating of temperature indicated in $\mathrm{Eq} .(16)$ is camied out first, and a radiation source function is calculated from

$$
u_{n}^{n *}=a\left(T^{n *}\right)^{4}=a\left(T^{n}+b^{-1} S \Delta t\right)^{4}
$$

then

$$
u_{r}^{n^{4}}=a T^{n^{4}}+4\left(T^{n}\right)^{3} b^{-1} S \Delta t+O\left((\Delta t)^{2}\right)=u_{r}^{n}+B S \Delta t+O\left(\Delta t^{2}\right)
$$


Thus $u_{n}^{n^{*}}$ calculated with the updated temperature $T^{n^{*}}$ from Eq. (16) agrees with $u_{n}^{n *}$ as defined by Eq. (5), to first onder in $\Delta t$. Therefore, we can safely do the temperature update (16) first and then calculate an emission source function for the radiation transfer part of the calculation afterward.

By our original assumption, $S$ includes the Compton energy exchange from the previous time cycle. To be completely consistent the energy contribution

$$
\left|\frac{\partial u_{m}}{\partial t}\right|_{C} \Delta t
$$

ought to be camied forwand to the next time cycle. However, it is obvious that the Compton temperature update can be considered to be made equally well at the end of the cument cycle as well as at the beginning of the next cycle. The only difference will be in the value of $b(T)$ employed. 3 The onder of the hydrodynamic update, whether made before or after the radiation update, is also inmaterial. The radiation update for Compton energy exchange and emission-absorption balance is made simultaneously at the end of the radiation transport calculation for the cycle.

\section{SUMMARY}

Let us assume for the sake of definiteness that the hydrodynamics is performed first during a given time cycle. Next, the temperature is updated by means of

One then calculates

$$
T^{n *}-T^{n}=b^{-1} \Delta t\left(\left.\frac{\partial u_{m}}{\partial t}\right|_{\text {Hydro }}\right.
$$

$$
u_{n}^{n *}=a\left(T^{n *}\right)^{4}
$$


Although one could also use

$$
u_{r}^{n^{*}}=a\left(T^{n}\right)^{4}+\beta\left(\frac{\partial u_{m}}{\partial t}\right)_{\text {Hydro }}
$$

if convenient. One also calculates

$$
\begin{aligned}
f & =\frac{\cdots}{1+\alpha \beta C \Delta t \sigma_{p}}, \\
\sigma_{v a} & =f \sigma_{v}, \\
\sigma_{v s} & =(I-f) \sigma_{v},
\end{aligned}
$$

The following transport equation is then solved with the radiation solution being advanced from $t^{n}$ to $t^{n+1}$

$$
\begin{aligned}
\frac{I}{c} \frac{\partial I_{v}}{\partial t}+\mu \frac{\partial I_{v}}{\partial x} & +\left(\sigma_{v a}+\sigma_{v s}+\sigma_{v}^{c}\right) I_{v}=\int \sigma^{c}\left(\mu^{\prime}, v^{\prime} \rightarrow \mu, v\right) I\left(\mu^{\prime}, v^{\prime}\right) \frac{v}{v^{\prime}} d v^{\prime} d \mu^{\prime} \\
& +\frac{I}{2}(I-f)\left(\sigma_{v} b_{v} / \sigma_{p}\right) \iint \sigma_{v^{\prime}} I_{v^{\prime}} d v^{\prime} d \mu^{\prime}+\frac{I}{2} f c \sigma_{v^{\prime}} b_{v} u_{r}^{n^{*}}
\end{aligned}
$$

The final temperature update is given by

$$
\begin{aligned}
& T^{n+1}=T^{n^{*}}+b^{-I} f\left\{\int_{t^{n}}^{t^{n+I}} d t \iint \sigma_{v} T_{v} d v d \mu-c \Delta t \sigma u_{p}^{n^{n *}}\right. \\
& +\int_{t^{n}}^{t^{n+1}} d t \int d \mu d v \int \sigma\left(\mu, v \rightarrow \mu^{\prime}, v^{\prime}\right) I(v, \mu) d v^{\prime} d \mu^{\prime} \\
& \left.-\int_{t^{n}}^{t^{n+1}} d t \int d \mu d v \int \sigma\left(\mu^{\prime}, v^{\prime} \rightarrow \mu, v\right) I\left(v^{\prime}, \mu^{\prime}\right) \frac{v}{v^{\prime}} d v^{\prime} d \mu^{\prime}\right\}
\end{aligned}
$$

where the first integral in Eq. (23) represents the cumulative effective absorption of radiation during a time cycle, and the second pair of integrals represents the accumulated energy transferred between radiation and matter through Compton scattering for the cycle. When a Compton 
scattering takes place, the photon's energy and frequency are appropriately changed, and the energy exchanged between radiation and matter is tallied for the appropriate zone.

If desired, the radiation calculation could be performed first, and the hydrodynamics last, in which case $u_{r}^{n}$ should replace $u_{r}^{n^{*}}, T^{n^{*}}$ replace $T^{n+1}$ and $T^{n}$ replace $T^{n^{*}}$ in Eq. (23). The hydrodynamics update would give

$$
T^{n+1}=T^{n^{*}}+b^{-1} \Delta t \frac{\partial u_{m}}{\partial t} \text { Hydro }
$$


1. UCRI-72924 "An Implicit Monte Carlo Scheme for Calculating Time and Frequency Dependent Nonlinear Radiation Transport."

2. It is possible, if desired, to take into account induced effects in a zeroth moment approximation as is now done in completely explicit calculations. The ensuing anguments used here will not be affected by this procedure.

3. From the point of view of effective energy conservation it is actually superior to work with the matter energy density variable $u_{m}$, to advance this quantity as a result of the various mechanisms of energy transfer and deposition, and then at the end of a time cycle to determine temperature as a function of $u_{m}$ by means of a table lookup. 
DISTRIBUTION

INTERNAL

C. Barnett

H. Bamett

R. Bentley

N. Byrne

E. Canfield

G. Chapline

A. Cole

G. Cooper

G. Corman

P. Crowley

D. Cummings

R. Debar

D. Eardley

F. Eby

P. Eltgroth

J. Fleck

E. Garelis

R. Gelinas

L. Germain

P. Giles

A. Goldberg

E. Goldberg

W. Grasberger

W. Grayson

D. $\mathrm{Hall}$

R. Howerton

C. Hendrickson

J. Hughes

N. Keeler

R. Kidder
J. LeBlanc

W. Loewe

W. Lokke

S. Maxon

M. May

R. McLean

L. Milton

P. Moulthrop

R. Nelson

W. Noh

J. Nuckolls

S. Perkins

E. Plechaty

A. Porter

D. Post

J. Ramus

W. Schulz

W. Shaw

C. Tarter

E. Teller

K. Trigger

P. Vajk

T. Wainwright

J. Wilson

A. Winslow

W. Zagotta

W. Zink

Technical Information Dept. ( 2 copies) Theoretical Files ( 3 copies) 